\title{
Espacios digitales y enfermedades raras
}

\author{
Laura Escobar Pérez, \\ Priscilla Avalos Sandoval y \\ Mara Medeiros
}

\section{Resumen}

La acidosis tubular renal (ATR) distal es una enfermedad que provoca retraso en el crecimiento óseo, , depósitos de calcio en el riñón, debilidad muscular y sordera. La ATR es una de las más de siete mil enfermedades raras conocidas en el mundo. Objetivo. Evaluar el impacto de los sitios www.funatim.org.mx y www.acidosistubular.unam.mx , su página de Facebook y un canal de Youtube, para contactar pacientes con el diagnóstico de ATR. Métodos. El análisis estadístico comprendió el diseño de la medición, la recolección de los datos y el análisis de la información. Resultados: Los medios digitales atrajeron a 3 pacientes con ATR y a cientos de familias con falsos diagnósticos. Predominó la participación de mujeres de la Ciudad de México y Jalisco; se tuvo alcance en 113 países. Algunas publicaciones en Facebook llegaron a más de 20,000 usuarios y a más de 70,000 en el sitio web durante un lapso de 4 años. Conclusiones: Las redes sociales conformaron una comunidad sólida que alertó los falsos diagnósticos y, a través de change.org, lograron que la Secretaría de Salud publicara la "Guía de práctica clínica para el diagnóstico y tratamiento de la acidosis tubular renal en pacientes pediátricos"..

Palabras clave: enfermedades raras, acidosis tubular renal, medios digitales, redes sociales.

\section{DIGITAL SPACES AND RARE DISEASES}

\begin{abstract}
Distal renal tubular acidosis (RTA) is a rare disease that provokes failure to thrive, calcium deposits in the kidney, muscular weakness and deafness. RTA is one of the more of seven thousand of rare diseases known around the world. Objective. Evaluate the web sites www.funatim.org.mx and www.acidosistubular. unam.mx, a facebook page of followers and a youtube channel, to contact patients with the diagnosis of RTA. Methods. The statistical analysis comprised the design of measurements, data collection and analysis. Results: Digital media attracted 3 patients with RTA and hundreds of families with false diagnosis. Most participants were women from Mexico City and Jalisco; digital publications was consulted by 113 countries. Some Facebook postings reached more than 20,000 users and more than 70,000 through the website during a period of 4 years. Conclusions: Social networks conformed a solid community to alert misdiagnosis. Through a request in change.org to the Ministry of Health, they achieved the publication: "Guide of clinical practices for the diagnosis and treatment of Renal Tubular Acidosis in pediatric patients".
\end{abstract}

Key words: rare diseases, renal tubular acidosis, digital media, social networks.

DOI: http://doi.org/10.22201/codeic.16076079e.2018.v19n5.a4 Recepción: 6/3/2018. Aprobación: 8/8/2018. 
"Espacios digitales y enfermedades raras"

\section{Laura Escobar Pérez}

Licenciada en Química, maestra en Ciencias Químicas y doctora en Investigación Biomédica Básica en la Universidad Nacional Autónoma de México. Es Profesora del Departamento de Fisiología de la Facultad de Medicina en la UNAM. Su línea de investigación es el estudio de canales iónicos en diferentes modelos celulares. En 1991 realizó una estancia postdoctoral en el Departamento de Neurobiología de la Escuela de Medicina de Harvard con el Dr. Roderick MacKinnon. Pertenece al Sistema Nacional de Investigadores (SNI). Fue becaria de la Royal Society y de la Fundación Carolina. Es socia fundadora de la "Fundación para la Acidosis Tubular Renal Infantil Mexicana" (FUNATIM). A través del sitio http://www.acidosistubular.unam.mx y el Facebook Acidosis Tubular Renal UNAM realiza actividades de divulgación en colaboración con profesionales de la salud. https://orcid.org/0000-0002-5142-8993.

\section{Priscilla Ávalos Sandoval}

pris@unam.mx

Es consultora en analítica digital con experiencia en proyectos para el sector de telecomunicaciones, financiero, seguros, retail y educación. Es ingeniera en Computación por la Universidad Nacional Autónoma de México. Durante 5 años fue académica de la Dirección de General de Cómputo y de Tecnologías de Información y Comunicación (DGTIC), donde participó en temas a favor del acceso abierto, redes sociales institucionales y visibilidad de los contenidos digitales universitarios. Ha sido instructora en analítica web y marketing digital con más de 7 años impartiendo cursos y diplomados en los que comparte su pasión por la medición. Es Coautora del libro Redes sociales digitales: nuevas prácticas para la construcción cultural, publicado por CONACULTA.

\section{Mara Medeiros Domingo}

medeiro.mara@gmail.com

Médico Cirujano, Facultad de Medicina UNAM. Especialista en Pediatría Médica y en Nefrología Pediátrica del HIMFG. Doctorado en Ciencias Biomédicas de la Facultad de Medicina de la UNAM. Post-doctorado en la Universidad de Cornell. Pertenece al Sistema Nacional de Investigadores. Colabora en el Hospital Infantil de México Federico Gómez (HIMFG). Investigadora en Ciencias Médicas y es responsable del Laboratorio de Investigación en Nefrología y Metabolismo Mineral. Es socia fundadora de la Fundación para la Acidosis Tubular Renal Infantil Mexicana AC y consejera de la Sociedad internacional de Nefrología Pediátrica y de la Asociación Latinoamericana de Nefrología Pediátrica. 


\section{Introducción}

Aproximadamente 7\% de la población mundial padece de alguna enfermedad rara, lo que equivale a cerca de 500 millones de personas. La legislación europea establece un límite de prevalencia menor de 5 casos por cada 10 mil personas para definir la rareza (FEDER, 2009). La Federación Mexicana de Enfermedades Raras (Femexer) estima que en México 7 millones de personas sufren de una de las 7 mil enfermedades raras existentes reconocidas por la Organización Mundial de la Salud (oms). El problema al que se enfrentan las familias con una enfermedad rara en países en desarrollo, es la falta de información confiable y accesible y de centros especializados donde atenderse; esto retrasa su diagnóstico y, por lo mismo, su tratamiento. En contraste, algunos países desarrollados tienen centros especializados y asociaciones dedicadas a pacientes con enfermedades raras y llevan a cabo investigación clínica y traslacional. La investigación traslacional consiste en llevar las preguntas que surgen en los hospitales a un laboratorio de investigación y, a la inversa, los hallazgos que se encuentran en el laboratorio, trasladarlos a la clínica para mejorar el tratamiento de los pacientes.

A pesar de los avances en la comprensión de varias enfermedades raras, la patogénesis (es decir, el origen y desarrollo) de muchas otras ha permanecido en el desconocimiento debido a que la mayoría de las enfermedades raras son complejas y tienen un origen genético; por otra parte, pocos médicos las conocen.

Respecto a las enfermedades renales, existen varias que son hereditarias en por lo menos 10\% de los pacientes con insuficiencia renal (Soliman et al, 2012); también hay factores genéticos que influyen en la progresión del daño crónico en las enfermedades renales adquiridas (Devuyst et al, 2014). Las enfermedades renales hereditarias tienen frecuencias variables; por ejemplo, la enfermedad poliquística del riñón autosómica dominante es la más frecuente y afecta a 1:1000 personas. En contraste, el resto de las enfermedades renales hereditarias son raras.

\section{Imposible objetivar de otro}

\section{modo las cosas mientras}

sigamos en el horizonte de las miradas heredadas por siglos de hacer ciencia sólo bajo ciertas perspectivas.
La dieta proteica occidental produce aproximadamente 1 miliequivalente $(\mathrm{mEq})$ de hidrogeniones $\left(\mathrm{H}^{+}\right)$por kg de peso corporal al día. En ausencia de un amortiguador en el plasma, la producción diaria de $\mathrm{H}^{+}$disminuiría el pH sistémico de 7.4 a 3 en tan sólo una hora, lo cual es incompatible con la vida. Por ello, el par ácido/base: $\mathrm{CO}_{2} /$ bicarbonato $\left(\mathrm{HCO}_{3}^{-}\right)$, constituye el amortiguador más importante porque mantiene el pH constante en el ambiente intra y extracelular.

El riñón filtra al día cerca de 180 litros de sangre y evita que perdamos el bicarbonato. El riñón expulsa en la orina la carga ácida proveniente de nuestra dieta proteica 
en forma de amonio; el ión amonio $\left(\mathrm{NH}_{4}^{+}\right)$es un ácido que se disocia en agua en amoniaco $\left(\mathrm{NH}_{3}\right)$. El par $\mathrm{NH}_{4}^{+} / \mathrm{NH}_{3}$ hace que el $\mathrm{pH}$ de la orina tenga valores entre 5 y 6 , lo cual permite que se deseche la carga ácida sin causarnos problemas.

La acidosis tubular renal (ATR) distal es una enfermedad en la que los pacientes no pueden eliminar el amonio en la orina, por lo que éste consume el bicarbonato de la sangre y se desarrolla acidosis metabólica con cloruro mayor al normal: pH menor de 7.4, niveles de bicarbonato menores de $16 \mathrm{mM}$; se forman depósitos de calcio en el riñón y disminuye el potasio en el plasma, lo que puede provocar parálisis muscular y arritmias. La respiración también se acelera y se desarrolla sordera nerviosa temprana o tardía (Escobar et al, 2013). La ATR tiene una prevalencia menor de 1 en un millón en la población en general y de 1 en 10 mil en sociedades con matrimonios consanguíneos (Elhayek et al, 2013). En el Reino Unido se documentó el primer estudio genético de ATR distal con 75 pacientes, la mayoría de origen turco (Stover et al, 2002); en España se han documentado 6 niños hispanos (Gil-Peña et al, 2014) y 8 casos de otros países (Gómez et al, 2016).

El grupo de investigación de la Dra. Laura Escobar descubrió una proteína en el riñón que transporta amonio (Carrisoza-Gaytan et al 2011). Fue entonces que se preguntaron: ¿qué pasa si esta proteína no funciona? Pues se acumularía amonio y, entonces, éste reaccionaría con el bicarbonato de la sangre, y eso es justo lo que sucede en los pacientes que tienen ATR distal. Así, la Dra. Escobar inició la búsqueda de pacientes con este padecimiento renal con el objeto de analizar sus genes. Pronto se percató que no existían casos documentados de ATR en la población mexicana. Para contactar familias con ATR publicó el blog www.funatim.org.mx (2010) y luego www.acidosistubular.unam.mx (2013).

El objetivo del trabajo fue evaluar el impacto que ha tenido la presencia digital de la Fundación para la Acidosis Tubular Renal Infantil Mexicana, A.C. (FUNATIM), en la difusión de información sobre ATR y diversos temas de salud; además de orientar a las familias que han sido erróneamente diagnosticadas con este síndrome en nuestro país.

Da click en la imagen para ver el video
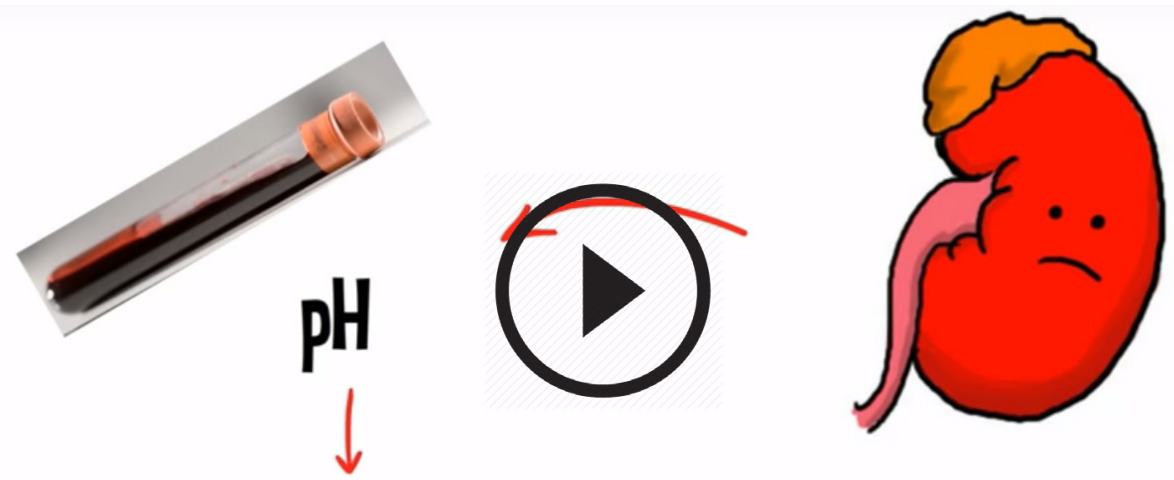
Da click en la imagen para ver el video

\section{Materiales y métodos}

Para el análisis estadístico se desarrolló un marco conceptual de 4 etapas: 1) diseño de la medición (revisión de métricas y dimensiones relacionadas con la visibilidad en medios digitales), 2) recolección de los datos (identificación de herramientas de analítica digital, desarrollo y aplicación de filtros de búsqueda), 3) análisis de la información (desarrollo de un análisis cuantitativo y cualitativo, orientado a destacar el impacto de los canales digitales) y 4) presentación de resultados (selección de gráficos representativos del análisis de acuerdo a las características de cada canal digital).

Se utilizaron como instrumentos: Google Trends (análisis orgánico), Google Analytics (analítica web), módulo nativo de estadísticas de Facebook y FanpageKarma (página de fans de Facebook) y el módulo nativo de Youtube Analytics (canal de Youtube). Para el análisis orgánico se revisó el periodo de 2004 a 2017, mientras que para el sitio web se analizaron los datos recopilados a partir del 31 de enero del 2013, fecha en que se comenzó a obtener estadística del sitio www.acidosistubular.unam.mx. Para la página de Facebook se analizó la información a partir del 30 de abril del 2013 y del canal Youtube a partir del 27 de abril del 2015. Para fines del presente trabajo se analizó la información hasta el 27 de enero del 2018.

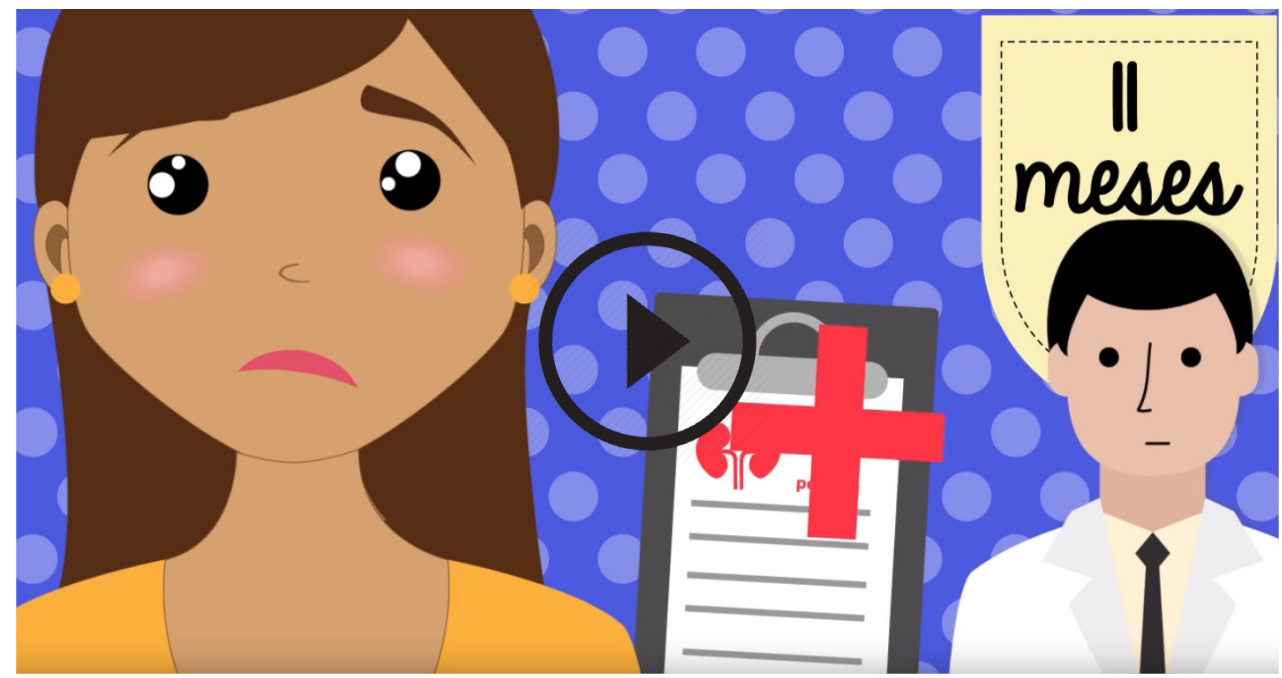

\section{Análisis de tendencias de búsqueda}

Para evaluar el impacto de la presencia digital, se analizó la tendencia del término "acidosis tubular renal" a través del tiempo, desde el motor de búsqueda Google. Se encontró que, a nivel internacional, el término ha sido buscado 
con mayor frecuencia por usuarios de Venezuela, México y Colombia (fig. 1a). A nivel nacional, las regiones de interés en donde se han realizado las consultas del término han sido: Ciudad de México, Jalisco, Puebla, Estado de México y Nuevo León (fig. 1 b).

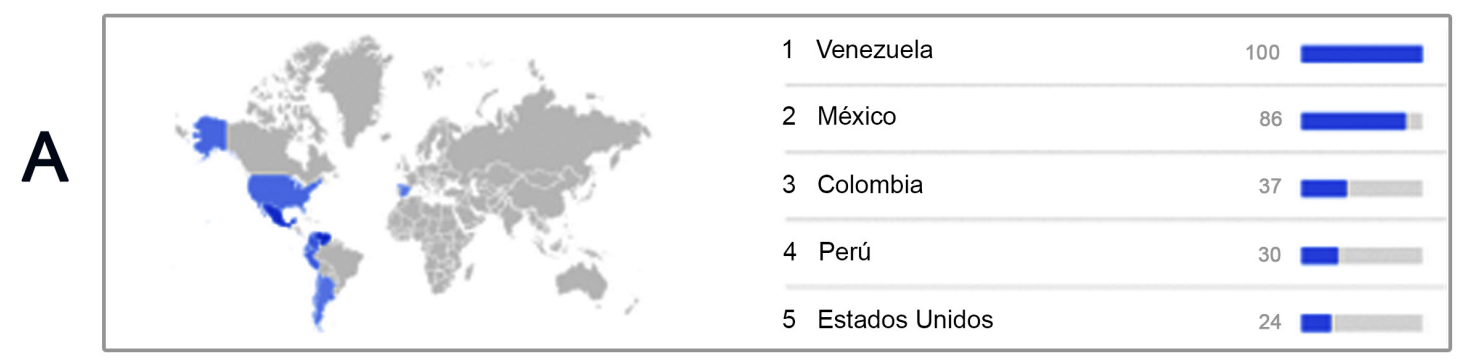

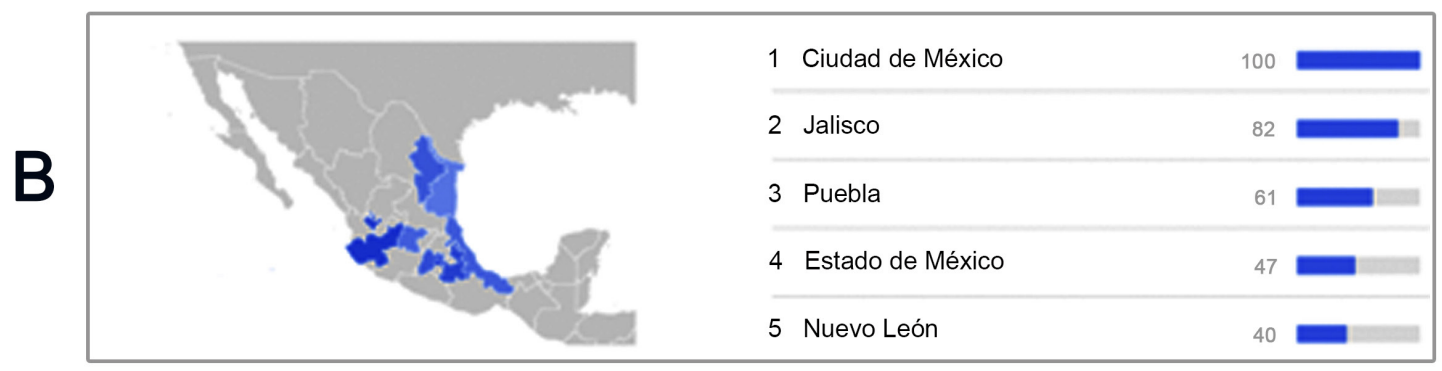

En el análisis de tendencia para el término "acidosis tubular renal", se observó que antes del 2005, las búsquedas del término eran más frecuentes; posterior a este periodo la tendencia disminuyó; sin embargo, para abril del 2011 y mayo del 2012 se presenta un nuevo interés en la búsqueda de dicho término, con la variante de: "acidosis tubular renal en niños" (fig. 2).

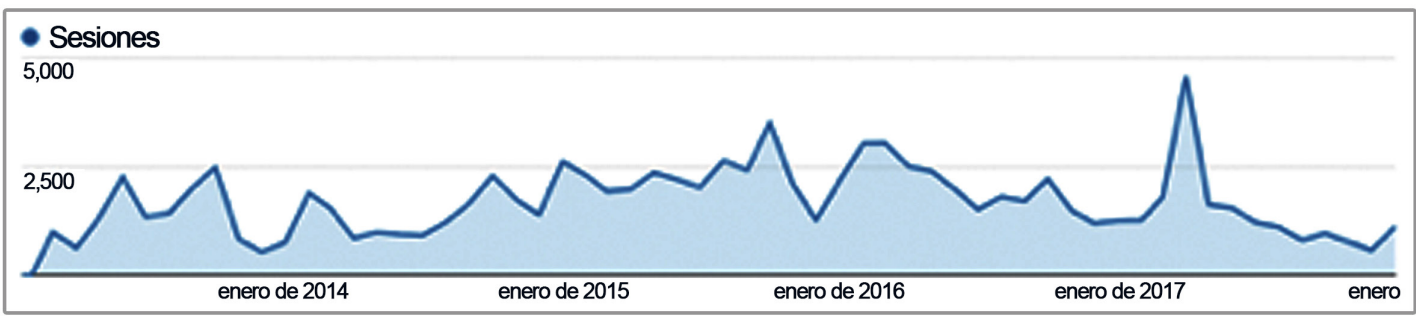

\section{Sitios web de la FUNATIM}

El primer canal de difusión de la Fundación fue el sitio web www.funatim.org.mx. Para el periodo en el que permaneció activo (2010-2013), el principal objetivo era poner a disposición del público interesado un canal informativo. La mayoría del contenido presentado eran textos y la comunicación con el público era a través de correo electrónico o de manera presencial.

Además de la puesta en línea del sitio web, se publicaron 2 artículos (Muñoz et al 2012; Escobar et al 2013) y 5 notas en medios externos (SIPSE, el blog 
de Acidosis Tubular Renal, Monografías, en el Boletín DGCS-UnAm y Mamá freelance). Con el objeto de analizar su impacto, se observó la cantidad de veces que los enlaces asociados a los artículos, notas y enlace del sitio actual, fueron compartidos. Además del sitio web (1,110 veces compartido) las notas: "Alertan de fraude con rara enfermedad", "Boletín UNAM-DGCS-514", y "Enfermedades de moda y su sobre diagnóstico" fueron las más compartidas en Facebook.

Con la evolución de la comunicación en medios digitales, y la necesidad de compartir contenidos multimedia, fue necesario actualizar la estructura del sitio de la funatim. Por tal motivo, el 21 de diciembre del 2012 se publicó una nueva versión alojada en el dominio institucional de la Universidad Nacional Autónoma de México (UNAM), en la dirección electrónica: www.acidosistubular.unam.mx. Cabe señalar que hasta marzo del 2013 se agregó un código de seguimiento para recolectar información estadística sobre la adquisición y comportamiento del sitio web, mediante el uso de Google Analytics. En el periodo comprendido entre el 4 de marzo de 2013 y el 27 de enero de 2018, se recibieron un total de 101,397 visitas, un promedio de 1,600 visitas mensuales (fig. 3). Durante marzo de 2017, el sitio alcanzó cerca de 4,589 visitas y entre junio-julio cerca de 9 mil. El sitio ha sido consultado en 113 países de los cuales 78\% de las visitas proviene de México.

Orgánico Pagado

\section{$9 \mathrm{~K}$}

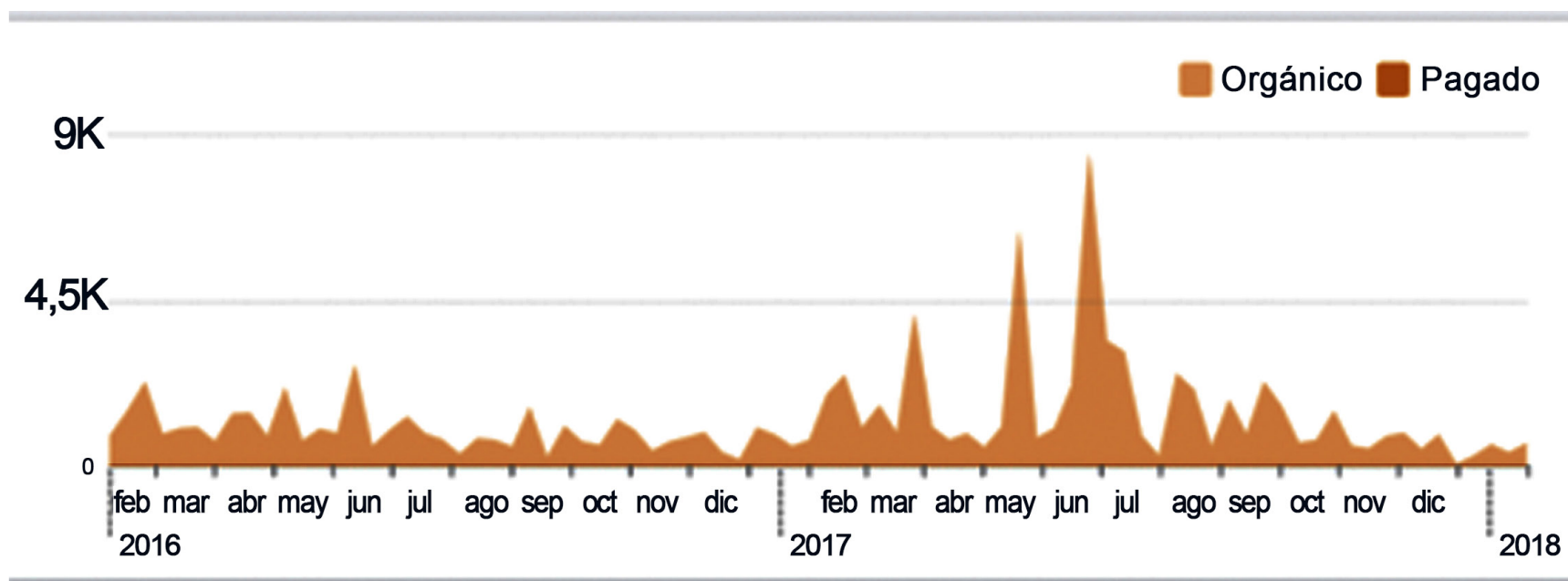

En la tabla se muestran los 10 países y las 10 ciudades de donde provienen la mayor cantidad de visitas. La Ciudad de México, el Estado de México y Jalisco son las que presentan mayor actividad.

\begin{tabular}{lcll}
\multicolumn{1}{c}{ País } & $\begin{array}{c}\text { Porcentaje de } \\
\text { visitas }\end{array}$ & \multicolumn{1}{c}{ Ciudad } & Porcentaje de visitas \\
\hline México & $78.57 \%$ & Ciudad de México & $44.91 \%$ \\
\hline España & $3.33 \%$ & Jalisco & $4.6 \%$ \\
\hline
\end{tabular}




\begin{tabular}{ll|ll}
\hline Colombia & $2.83 \%$ & Nuevo León & $1.63 \%$ \\
\hline Perú & $2.59 \%$ & Estado de México & $1.51 \%$ \\
\hline Estados Unidos & $2.13 \%$ & Lima Región & $1.14 \%$ \\
\hline Argentina & $2.01 \%$ & Puebla & $1.13 \%$ \\
\hline Venezuela & $1.63 \%$ & Bogotá & $1.11 \%$ \\
\hline Chile & $1.12 \%$ & Querétaro & $0.98 \%$ \\
\hline Ecuador & $0.69 \%$ & Buenos Aires & $0.97 \%$ \\
\hline Guatemala & $0.52 \%$ & Yucatán & $0.82 \%$
\end{tabular}

Tabla. Principales países y ciudades de donde provienen las visitas al sitio web de FUNATIM (periodo 31 enero 2013 - 31 julio 2017). Fuente: Google Analytics.

En cifras del sitio web se encontró que $73 \%$ de los visitantes que lo consultan son mujeres mientras que $27 \%$ son hombres, en su mayoría entre los 25 y 34 años. Éste se ha convertido en un espacio de contenidos relacionados con la ATR, que está indexado y posicionado en buscadores en México. Los contenidos más consultados por los visitantes son: “Qué es el ATR?" (con 49,051 visitas; 31.73\%) y “'A quién consultar?" (con 12,170 visitas; 7.87\%).

\section{Principales fuentes de tráfico}

La mayoría de los usuarios que llegan al sitio web lo hace a partir de búsquedas en Google, esto se debe al buen posicionamiento del dominio unam.mx al que se encuentra asociado el sitio de la funAtim. Del total, 56.54\% llega al sitio web a partir de búsquedas en Google organic search, seguido por los usuarios que ingresan directamente colocando la dirección electrónica del sitio en el navegador Direct (18.90\%), la tercera fuente y la más importante de esta investigación, pues se refiere a los visitantes que llegaron al sitio de la fUNATIM a partir de alguna red social (12.72\%) y finalmente referral (11.82\%) que se refiere a las visitas al sitio a través de un vínculo o referencia en sitios web externos (sitios de noticias, blogs, portal UnAM, entre otros).

\section{Las redes sociales como fuente de adquisición}

Al realizar el análisis de las redes sociales que actúan como medio de adquisición al sitio web de la FUNATIM, se encontró que de las 12,915 sesiones que vienen de Social, 93,16\% provienen de Facebook donde la URL más compartida ha sido la página de inicio del sitio web. 


\section{Página de seguidores (fans) de Facebook}

La página de fans de Facebook ha sido un canal de vinculación importante para la fUNATIM, pues fue a través de este canal que en agosto del 2013 cerca de 1,200 familias solicitaron, en el sitio Change.org a la Secretaría de Salud Pública (SSP) elaborar una guía de diagnóstico de ATR. En un hecho insólito, en enero del 2017 la ssp publicó la "Guía de prácticas clínicas para el diagnóstico y tratamiento de la atr en pacientes pediátricos".

La página de fans Acidosis Tubular Renal-unAm creada en abril del 2013 tuvo, hasta enero de 2018, 2,551 Me gusta. Acorde a la comunidad digital, la página de fans también se conforma en su mayoría por mujeres (80\%), principalmente entre los 25 y 34 años, provenientes de México (Ciudad de México y Guadalajara); dato que coincide con las búsquedas orgánicas y el análisis geográfico del sitio. En promedio se realizan 3 publicaciones diarias, con un nivel de servicio de 86\%; es decir, que en menos de 14 horas se brinda una respuesta a las preguntas de los usuarios. La labor de publicación se ha realizado todos los días de la semana, abarcando en promedio un horario de las 8:00 a las 23:30 horas. Los días de mayor interacción son los lunes por la noche y los jueves por la mañana.

Con la finalidad de mantener informada a la comunidad, se comparten notas, contenidos y consejos relacionados con la ATR, nutrición infantil y temas de salud en general.

La gestión de la página de fans se ha basado en la estrategia de contenidos especializada en el tema central: ATR, y sin el uso de anuncios pagados. De esta forma, se ha conseguido el crecimiento paulatino de manera orgánica (fig. 3).

Entre las publicaciones que destacan por la respuesta positiva de la comunidad se encuentran las siguientes:

1. "Estudio de investigación clínica que se realizó en el Hospital Centro Médico Imss la Raza", con un alcance de 20,557 personas (https://bit. ly/2MaQafz).

2. "Guía de prácticas clínicas para el diagnóstico y tratamiento de ATR en pacientes pediátricos", con un alcance de 15,641 personas (https://bit.ly/2MbuxMq).

I. "Los dos primeros años de vida son críticos para el crecimiento de los niños", con un alcance de 12,352 personas (https://bit. ly/2nDW62h).

Los videos publicados en Facebook que han sido más consultados son:

3. "Acidosis Tubular Renal (material original)", con un alcance de 3,355 personas en el canal de la funatim. 
4. "Córdoba realiza el primer trasplante de hígado entre una abuela y su nieto", con un alcance de 2,685 personas.

5. "Testigo daño renal infantil, entrevista a la Dra. Laura Escobar para el programa 'Creadores Universitarios' por Foro TV', con un alcance de 1,629 personas.

\section{Canal de YouTube}

El canal de funatim unam en YouTube fue creado el 23 de abril de 2015. Desde su inicio hasta el 27 de enero de 2018, la gestión del canal ha acumulado 635 suscriptores y 87 videos publicados, los cuales han sido visualizados 86,617 veces y compartidos 1,074 veces. Respecto a la retención de la audiencia, un usuario promedio del canal visualiza 5 minutos 49 segundos de material producido por el canal de la funATIM. Al igual que los demográficos del sitio web y la página de fans de Facebook, la mayoría de la audiencia del canal de la funATIM es del género femenino (58\%) de 25 a 34 años, pero los hombres de la misma edad son los que más tiempo permanecen en el canal. Los usuarios que han consultado los videos de la funATIM provienen principalmente de Latinoamérica: México (39\%), Perú (11\%), Colombia (9.4\%), Argentina (8.4\%), Venezuela (4.9\%) y Chile (4.4\%). De esta muestra, $95 \%$ ha consultado los videos a través de la plataforma Youtube.com y sólo $5 \%$ a través de otros sitios externos donde se han insertado los videos. La mayoría de los usuarios (50\%) que han visualizado los videos del canal ha sido porque los contenidos les han aparecido como "videos relacionados". La segunda fuente de tráfico a los contenidos ha sido a partir del uso del buscador de Youtube (24\%), lo cual indica que los contenidos cuentan con el etiquetado suficiente para ser encontrados con facilidad a través de la plataforma de Youtube.

El contenido publicado en el canal de Youtube pasa por un proceso de curaduría, con la intención de presentar contenidos de calidad para la comunidad interesada. Los materiales se componen de animaciones, conferencias y grabaciones de eventos organizados por la propia Fundación. Los usuarios del canal consumen en promedio $22 \%$ de la duración total de los vídeos publicados por la funatim en Youtube. Cabe destacar que la mayoría de los videos publicados tienen una duración mayor a 30 minutos. Si se considera que el tema tratado es especializado y que los consumidores promedio prefieren consultar vídeos de corta duración en Youtube, es relevante que los usuarios pasen en promedio 5.49 minutos visualizando el contenido.

Con base en la lista de los videos con mayor número de visualizaciones y su asociación a la cantidad de Me gusta (635) y No me gusta (38), se puede concluir que el contenido es aceptado positivamente por la comunidad. El canal de la funatim ha recibido un total de 50 comentarios, de los cuales 10 han sido 
asociados a un vídeo que explica el caso de un niño que sufrió las consecuencias de un falso diagnóstico de ATR. Este video es uno de los de menor duración (3.58 min) en el canal.

Posterior a la publicación del sitio web, y la actividad que se ha ido generando en los canales de la FUNATIM, han surgido comunidades activas que fomentan la difusión y participación del público interesado, entre las que destacan el grupo privado: "Creciendo juntos (Acidosis Tubular Renal)" con una comunidad de 1,175 miembros y el grupo público de "Acidosis Tubular Renal (El diagnóstico infundado)" con 751 miembros. Ambas comunidades surgieron por madres de niños atendidos por la fUNATIM y que, en busca de continuar con la labor de difusión y prevenir los casos de falsos diagnósticos, brindan apoyo y asesoría a padres de niños a los que les han diagnosticado ATR. Al analizar el tipo de contenido compartido en estos grupos, se identificó que en su mayoría son testimonios de la propia comunidad, donde también se comparten materiales informativos, eventos y contenidos generados por la FUNATIM.

\section{Conclusiones}

Los canales digitales gestionados con responsabilidad son una fuente de conocimiento y experiencias que puede almacenarse para informar atemporalmente a la población, independientemente de que el tema tratado sea sumamente especializado y afecte solo a un reducido sector de la población.

El sitio de la fUnATIM se encuentra posicionado en los principales motores de búsqueda, al realizar consultas con el término "Acidosis Tubular Renal". Asimismo, el sitio ha sido fuente de referencia a través de revistas, boletines de la unAm, así como blogs y sitios web externos.

Las redes sociales permiten convocar a personas y formar comunidades sólidas que se mantienen informadas, se identifican y apoyan entre sí. Las redes sociales que ayudaron a la difusión de la labor de la funATIm tuvieron la capacidad de lograr acuerdos con la Secretaría de Salud, principal órgano gubernamental, para elaborar la "Guía de prácticas clínicas para el diagnóstico y tratamiento de la ATR en pacientes pediátricos".

\section{REFERENCIAS}

* Acidosis tubular renal (El diagnóstico infundado). Disponible en: https://www.facebook.com/groups/594369127252927/?fref=ts.

- Blog Acidosis Tubular Renal - ATR (enero, 2012). http://atr-acidosistubularrenal. blogspot.mx. 
* Boletín unam-dgcs-514 (31 agosto, 2011). Consultado el 11 de agosto 2018. Disponible en: http://www.dgcs.unam.mx/boletin/bdboletin/2011 514.html.

* Carrisoza-Gaytán, R., Rangel, C., Saldaña-Meyer, R., Salvador, C., Escalona, C., Satlin, L. M., et al. The hyperpolarization-activated cyclic nucleotide-gated HCN2 channel transports ammonium in the distal nephron. Kidney Int 2011; 80:832-40. https://doi.org/10.1038/ki.2011.230.

* CENETEC. Diagnóstico y Tratamiento de la Acidosis Tubular Renal en pacientes pediátricos. Guía de Evidencias y recomendaciones, México, Centro Nacional de Excelencia Tecnológica en Salud, Secretaria de Salud; 2016 (consulta 11 de agosto 2018). Disponible en: http://cenetec-difusion.com/CMGPC/SS-255-16/ER.pdf.

* Creciendo juntos acidosis tubular renal. Disponible en: https://www.facebook. com/groups/creciendojuntosatr/?fref=ts.

- Devuyst, O., Knoers, NVAM, Remuzzi, G. y Schaefer, F. (2014). Rare inherited kidney diseases: challenges, opportunities and perspectives. Lancet; 383:1844-1859. https://doi.org/10.1016/S0140-6736(14)60659-0.

* Elhayek, D., Perez de Nanclares, G., Chouchane, S., Hamani, S., Mlika, A., Troudi, M., et al. (2013). Molecular diagnosis of distal renal tubular acidosis in Tunisian patients: proposed algorithm for Northern Africa populations for the ATP6V1B1, ATP6V0A4 and SCL4A1 genes. BMC Med Genet; 14:119. Dol: https://doi. org/10.1186/1471-2350-14-119.

* Escobar-Pérez, L. I., Mejía, N., Gil, H. y Santos, F. (2013). La acidosis tubular renal distal (ATRD): una enfermedad hereditaria rara en la que no se puede eliminar la carga ácida. Nefrología 33:289-96. Dol: https://doi.org/10.3265/Nefrologia. pre2012.Oct.11592.

- FEDER (2009). Estudio sobre situación de necesidades socio sanitarias de las personas con enfermedades raras en España. Estudio ENSERio [edición electrónica] https:// enfermedades-raras.org/images/stories/documentos/Estudio ENSERio.pdf.

* Gil-Peña, H., Mejía, N. y Santos, F. (2014). Renal tubular acidosis. J Pediatr ;164: 691-98. Dol: https://doi.org/10.1016/j.jpeds.2013.10.085.

* Gómez, J., Gil-Peña, H., Santos, F., Coto, E., Arango, A., Hernández, O., et al. (2016). Primary distal renal tubular acidosis: novel findings in patients studied by next-generation sequencing. Pediatric Research; 79: 496-501. Dol: https://doi. org/10.1038/pr.2015.243.

* Gomez-Salas, D. (2011). Detección y detención de los daños de la acidosis tubular renal. Consultado el 11 de agosto 2018. http://blogs.monografias.com/ noticiencias/2011/09/01/deteccion-y-detencion-de-los-danos-de-la-acidosistubular-renal/.

* Guerra-Hernandez, N., Matos-Martínez, M., Ordaz-López, K., Camargo-Muñiz, D., Medeiros, M., Escobar-Pérez, L. (2014). Clinical features and progress of mexican patients with distal renal tubular acidosis. Rev Invest Clin 2014; 66(5)386-392. http://www.medigraphic.com/pdfs/revinvcli/nn-2014/nn145b.pdf. 
* Muñoz-Arizpe, R., Escobar, L., Medeiros, M. (2012). Over-diagnosis of renal tubular acidosis in Mexico. Rev Invest Clin; 64:399-401. http://www.medigraphic.com/ pdfs/revinvcli/nn-2012/nn124j.pdf.

* Muñoz-Porras, V. (2011). Enfermedades de moda y su sobre diagnóstico. Consultado el 9 de agosto 2018. Disponible en: http://www.mama-freelance. com/2011/05/06/enfermedades-de-moda-y-su-sobre-diagnstico-el-caso-particular-del-atr/.

- Soliman, N. A. (2012). Orphan kidney diseases. Nephron Clin Pract; 120: c194-9. DoI: https://doi.org/10.1159/000339785.

Stover, E. H., Borthwick, K. J., Bavalia, C., Eady, N., Fritz, D. M., Rungroj, N., et al. (2002). Novel ATP6V1B1 and ATP6V0A4 mutations in autosomal recessive distal renal tubular acidosis with new evidence for hearing loss. J Med Genet; 39: 796803. https://jmg.bmj.com/content/39/11/796.

* Valdéz, B. (2012). Alertan de fraude con rara enfermedad (29 noviembre, 2012). https://sipse.com/ciencia-y-salud/alertan-de-fraude-con-rara-enfermedad-3064.html.

* Yañez, G. (2013). Petición change.org. Secretaria de Salud México: Instaurar un protocolo oficial para el correcto diagnóstico de la "ATR". Consultado el 11 de agosto 2018. https://bit.ly/2MGzpFj.

\section{Cómo citar este artículo}

- Escobar Pérez, Laura, Avalos Sandoval, Priscilla y Medeiros Domingo, Mara (2018). Espacios digitales y enfermedades raras. Revista Digital Universitaria (RDU). Vol. 19, núm. 5 mayo-junio. DOI: http://doi.org/10.22201/codeic.16076079e.2018.v19n5.a4. 